\title{
Paper Straws: An Investigation into Surface Modification and Hydrophobization of Cellulose
}

$10.17975 /$ sfi-2020-007

\section{Norbert Banyi and Jordan Hassett}

University of British Columbia

ABSTRACT: In order to improve the quality of paper straws, experiments involving the hydrophobization of paper, in a silylation reaction with chloro(dimethyl)octadecylsilane using various solvents, were conducted. The ImageJ program was used to quantify hydrophobicity by calculating the contact angle between a water droplet and a small piece of paper, which were compared between treatment groups as well as with untreated paper and plastic straws. Samples were exposed to a variety of liquids in one-hour periods for a total of six hours. After each hour, contact angle measurements were taken. Results suggested that hydrophobicity declines with time due to leaching of silanol from the treated paper. Contact angles between water droplets and the treated paper remained larger than that of untreated paper straws throughout testing, indicating higher hydrophobicity. Furthermore, samples that were silylated using dioxane as a solvent were better able to maintain hydrophobicity than samples silylated using toluene as a solvent.

KEYWORDS: hydrophobization, silanization, surface modification, paper straws, biodegradable

\section{INTRODUCTION}

In 2013, over 299 million tons of plastics were produced, with plastic consumption in more prosperous nations, such as those in Western Europe, exceeding $200 \mathrm{lb}$ per person per year $(1,2)$. As plastic waste accumulates on the planet, there has been a shift away from single-use plastic products towards sustainable alternatives. One notable case is the plastic straw and its paper-based replacement. Having a paper-based straw allows for the convenience of a single-use straw, while also mitigating the harmful impact of plastic accumulation on animals and their ecosystems. However, current paper straw models readily absorb water, meaning that their structural integrity degrades considerably with time. Thus, despite being a more environmentally friendly option, the substandard quality of current paper straw models makes them an unfeasible alternative for plastic straws. This investigation will explore a possible method for enhancing the hydrophobicity of paper in order to make it a more appealing and viable replacement for plastic straws.

The configuration and overall polarity of a molecule plays a role in determining whether it is hydrophobic or hydrophilic. Cellulose, the primary constituent of paper, is a polymer with polar glucose residues (Figure 1), and as such has hydrophilic interactions with water (3). In order to make paper more hydrophobic, the chemical structure may be altered to decrease the polarity by covalently attaching non-polar moieties, such as hydrocarbon chains, to the polar hydroxyl groups. A typical hydrocarbon consists of a linear alkyl chain made up of carbon and hydrogen, making it very non-polar. As the length of the chain increases, the impact of any polar substituents becomes negligible to the overall hydrophobicity of the molecule (as seen in Table 1). Therefore, using large hydrocarbons to derivatize the cellulose in paper straws would lower the overall polarity and increase hydrophobicity.

Chloro(dimethyl)octadecylsilane $\left(\mathrm{C}_{20} \mathrm{H}_{43} \mathrm{SiCl}\right)$ has a hydrocarbon chain of 18 carbons, making it both sufficiently non-polar, and large enough to alter the hydroaffinity of the surface of paper. When it binds to the cellulose molecules, the chlorine is removed from the molecule and the silicon covalently bonds with an oxygen atom from one of the hydroxyls on the cellulose (Figure 1). This silicon-oxygen bond is among the strongest of common covalent bonds, with an average bond energy of $452 \mathrm{~kJ} / \mathrm{mol}$, making it ideal for binding the hydrophobic hydrocarbon chain to cellulose (5). 
Table 1. Solubility of select alcohols in water (Based on data from reference 4).

\begin{tabular}{|l|l|l|}
\hline Compound & Formula & Solubility in $\mathrm{H}_{2} \mathrm{O}\left(\mathrm{mol} / 100 \mathrm{H} \mathrm{H}_{2} \mathrm{O}\right.$ at $\left.20^{\circ} \mathrm{C}\right)$ \\
\hline Ethanol & $\mathrm{CH}_{3} \mathrm{CH} 2 \mathrm{OH}$ & Completely Miscible \\
\hline Butanol & $\mathrm{CH}_{3} \mathrm{CH}_{2} \mathrm{CH}_{2} \mathrm{CH}_{2} \mathrm{OH}$ & 0.11 \\
\hline Pentanol & $\mathrm{CH}_{3} \mathrm{CH}_{2} \mathrm{CH}_{2} \mathrm{CH}_{2} \mathrm{CH}_{2} \mathrm{OH}$ & 0.030 \\
\hline Hexanol & $\mathrm{CH}_{3} \mathrm{CH}_{2} \mathrm{CH}_{2} \mathrm{CH}_{2} \mathrm{CH}_{2} \mathrm{CH}_{2} \mathrm{OH}$ & 0.0058 \\
\hline Heptanol & $\mathrm{CH}_{3} \mathrm{CH}_{2} \mathrm{CH}_{2} \mathrm{CH}_{2} \mathrm{CH}_{2} \mathrm{CH}_{2} \mathrm{CH}_{2} \mathrm{OH}$ & 0.0008 \\
\hline
\end{tabular}

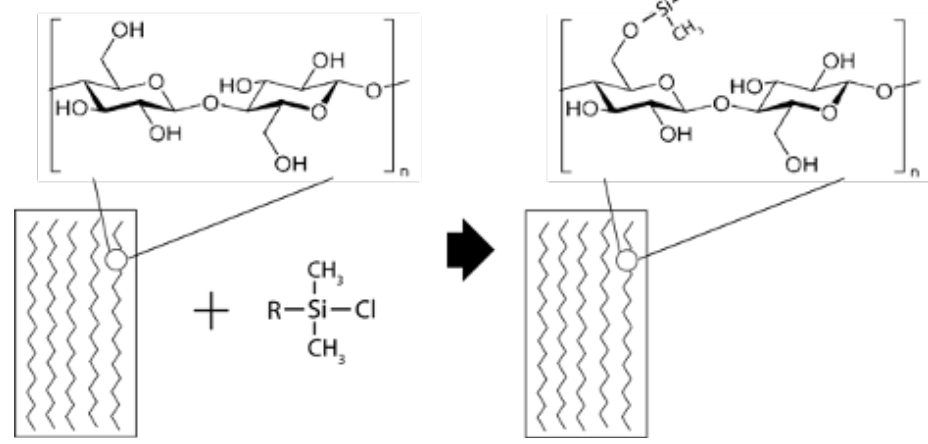

Figure 1. Silylation of ceullulose through the formation of a silicon oxygen bond between chloro(dimethyl)octadecylsilane and a 6 hydroxyl of the glucose residues $\left(\mathrm{R}=\mathrm{C}_{1} 8 \mathrm{H}_{37}\right)$. All hydroxyls are reactive, but due to the bulky nature of the silane, the primary hydroxyl is more reactive than the secondary. (6)

Determining the contact angle between the water droplet and the hydrophobized cellulose surface provides a method for quantifying hydrophobicity (7). Contact angles are measured by generating a line tangent to the water droplet, starting at the contact point between the droplet and the surface, and measuring the angle between the surface and the tangent (Figure 2).

\section{METHODOLOGY}

\section{Paper Preparation}

The preparation procedure was developed based on a previous study reviewing the preparation of reverse-phase silica gel (8). Whatman ${ }^{\circledR}$ qualitative filter paper is made purely of cellulose (9), so it was used instead of premade paper straws in order to avoid any possible reactions that

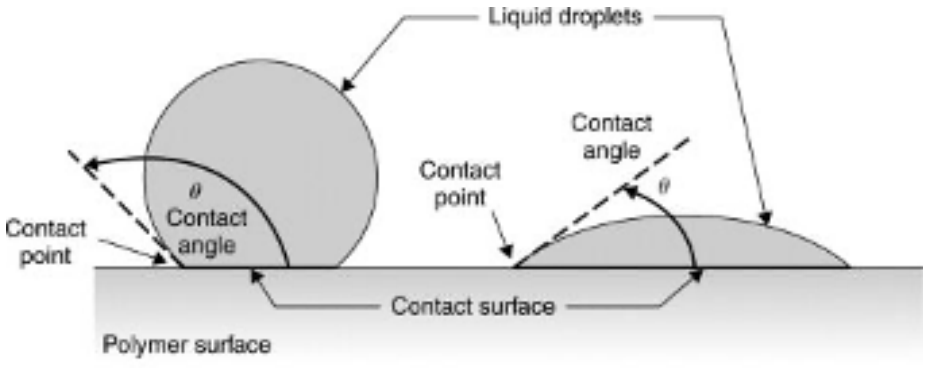

(a)

(b)

Figure 2. Demonstration of contact angle measured for (a) a hydrophobic surface, and (b) a hydrophilic surface. (Figure taken from (8)).

may occur through the use of treated papers. The filter paper was cut into $252 \times 1 \mathrm{~cm}^{2}$ squares and divided into six groups, each correlating to a different treatment as illustrated in Figure 3. Treatments used $5 \mathrm{~mL}$ of the chlorosilane reagent as well as $20 \mathrm{~mL}$ of one of three different solvents dried with a 4 Å molecular sieve: toluene, dimethylformamide, and dioxane. Groups were designated based on treatment conditions, as demonstrated in Figure 3: (A) dry dioxane + chlorosilane (DSS), (B) dry dioxane (DS), (C) dry toluene + chlorosilane (TSS), (D) dry toluene (TS), (E) dry dimethylformamide + chlorosilane (FSS) and (F) dry dimethylformamide (FS). Mixtures were combined with 95\% chloro(dimethyl)octadecylsilane (acquired from Sigma Aldrich (10)) in Erlenmeyer flasks, dried using an autoclave and sealed using self-healing injection stoppers. Reagents were injected under argon gas to eliminate contact with water vapour and shaken using a platform shaker for 1 hour at $37^{\circ} \mathrm{C}$ and $180 \mathrm{rpm}$, then at room temperature for another 20 hours at 95 rpm. Paper samples were then removed from the mixtures and washed three times with 


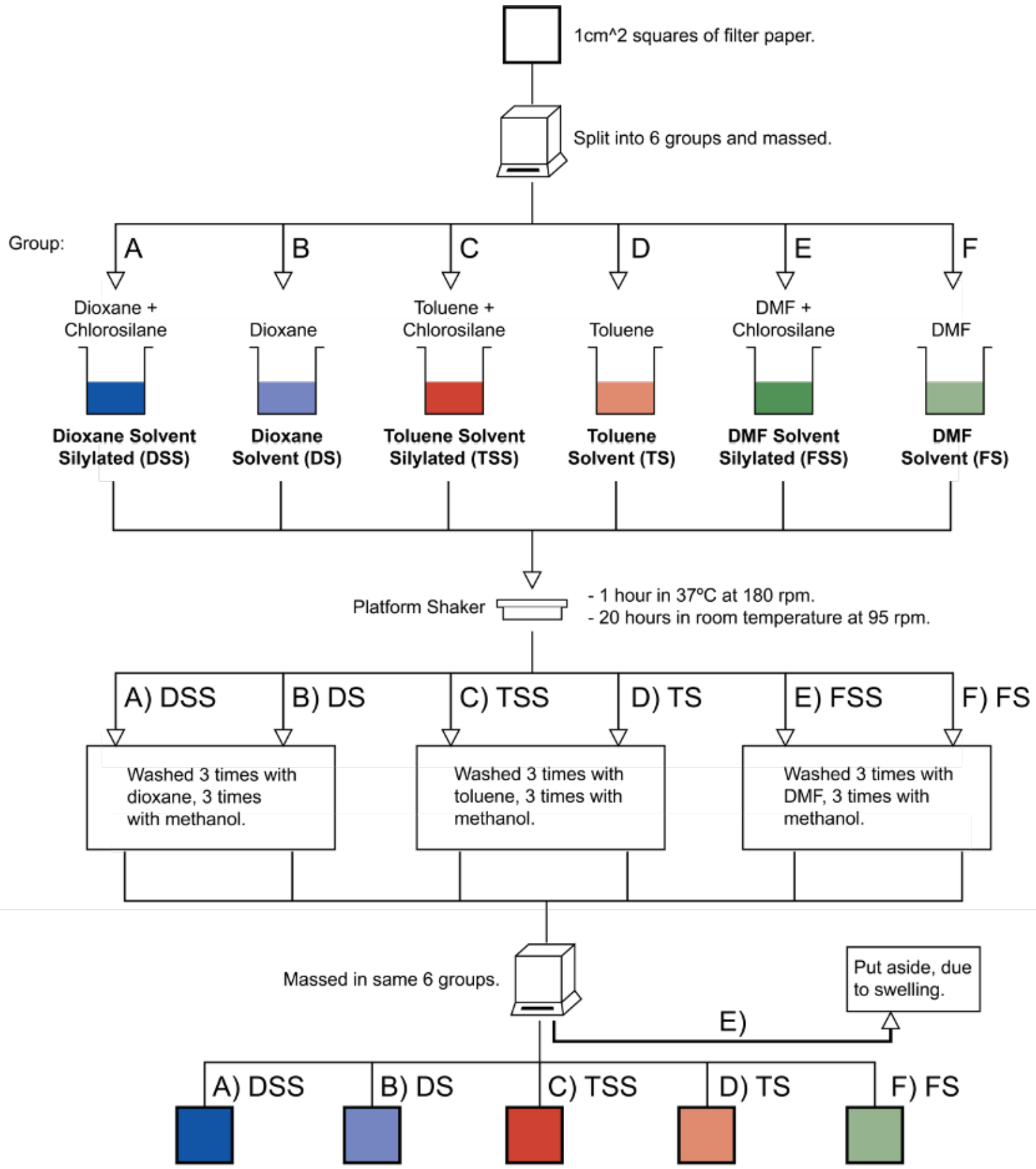

Figure 3. Procedure for the treatment of paper samples; $252 \times 1 \mathrm{~cm}^{2}$ samples of Whatman ${ }^{\circledR}$ cellulose filters were massed in six groups. Each group was submerged into one of three different solvents with and without reagent and placed on a platform shaker. The platform shaker was set to $180 \mathrm{rpm}$ for 1 hour at $37^{\circ} \mathrm{C}$, followed by 20 hours in room temperature at $95 \mathrm{rpm}$. Samples were washed 4 times with their respective solvents and 3 times with methanol. All samples were massed again, but the papers treated with dimethylformamide (DMF) and chlorosilane became swollen during the procedure so they were put aside. 


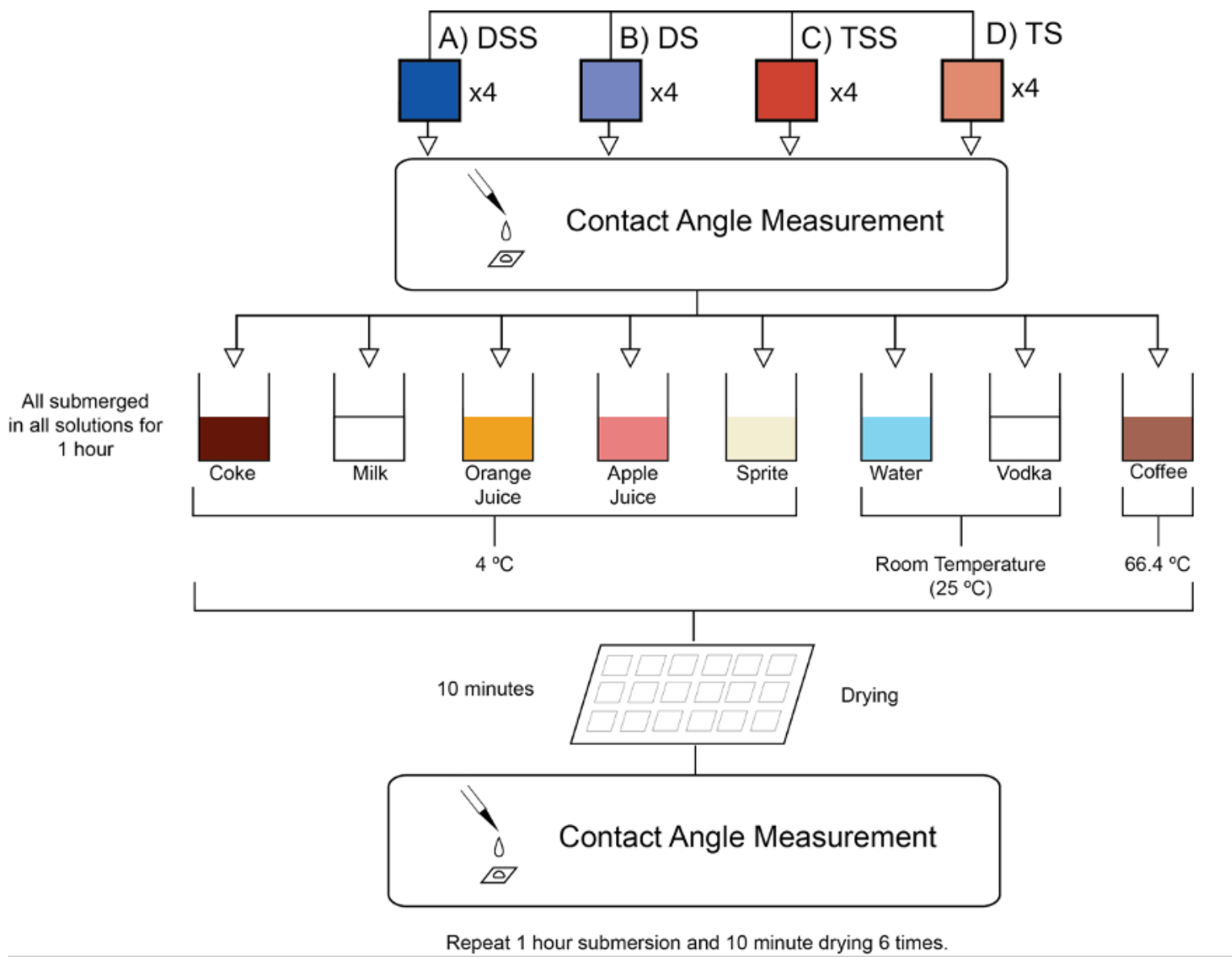

Figure 4. Outline of how primary testing had been conducted; DMF samples were not included due to swelling. Four samples from the DSS, DS, TSS, TS groups were exposed to each of the above solutions for 1 hour, dried for 10 minutes, and used to measure respective contact angles. Using the same samples, this procedure was repeated for 6 hours of exposure.

their corresponding reaction solvent, then three times with methanol to remove all of the solvent. All samples were weighed both before and after silylation with an analytical balance.

\section{Hydrophobicity Testing and Analysis}

DSS, DS, TSS, and TS samples were tested, then compared between treatment groups. Additionally, the results were compared with those of tests we conducted using commercial paper straws and plastic straws, which are often made from polypropylene (11). Samples treated with dimethylformamide were excluded from testing as justified in the appendix.

Droplets of water smaller than $10 \mu \mathrm{L}$ are small enough that the effect of gravity on static contact angle values becomes negligible (12). As such, $5 \mu \mathrm{L}$ water droplets were administered onto each sample from a controlled height of 1 $\pm 0.17 \mathrm{~cm}$ using a micropipette and recorded as a video to be reviewed later.
Initial contact angle measurements were taken for all paper samples following silylation. Groups were designated for each solvent treatment used. Each group consisted of four paper samples, which were then placed into the drinks as shown in Figure 4. After every hour, the samples were removed and dried for 10 minutes, then the contact angle was measured before returning each sample to its respective beverage. This was done six times for a total of 6 hours of submersion.

The long-term hydrophobicity between DSS and TSS samples was assessed by looking at the difference in contact angle after allowing a $5 \mu \mathrm{L}$ droplet to sit on each sample for 30 minutes. The change in mass of DSS and TSS samples after 6 hours of exposure to Coca Cola and water) were investigated by massing samples before and after testing.

Videos were analyzed using ImageJ, a Java-based image processing program developed at the National Institute of Health and the Laboratory for Optical and Computational 


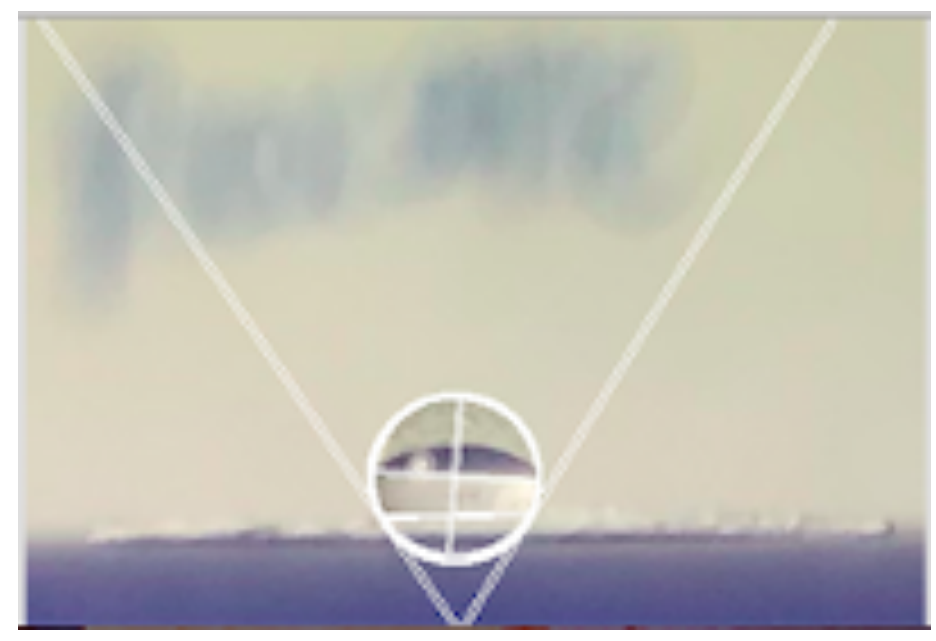

Figure 5. Circularly fitted $5 \mu \mathrm{l}$ droplet of water using Image software.

Instrumentation. Using contact points, the program measured the contact angle between the sample and water by generating a circle fit to the drop (Figure 5). Though the program itself has error, the uncertainty in identifying the contact points with precision and accuracy is considerably greater. Error was accounted for by taking three measurements of contact angle for each of the four replicates, then taking the standard deviation between the four replicates. Single variable Anova tests were used to compare between the treated paper straws and the current paper straws.

\section{RESULTS}

Results show a decline in contact angle in both DSS and TSS samples throughout the 6-hour testing period for all beverages being tested (Figure 6). This decline was most evident in coffee and Coca Cola. Overall, contact angles for TSS samples declined much faster than DSS samples in every drink except for water, where angles remained approximately equal throughout the testing period (vodka: $p=0.045$, water: $p<0.001$, coffee: $p<0.001$, milk: $p<0.001$, sprite: $p=0.028$, orange juice: $p=0.029$, apple juice: $p=0.018$, Coca-Cola, $p=0.018$ ). DSS samples maintained significantly higher contact angles than untreated paper samples for all drinks throughout the entire experiment. Paper samples were weighed before and after treatment to show how much silanol was added. DSS samples showed a significantly higher average increase in mass $(0.225 \mathrm{mg})$ than TSS samples (0.1905 mg) after silylation (Table 2). Interestingly, samples showed non-significant change in mass and contact angle after exposure to water for 6 hours, but showed an increase in mass, combined with a significantly lower contact angle after exposure to Coca Cola for 6 hours (Table 3). In addition, TSS samples showed a significantly greater decline in contact angle overall than DSS samples after having a water droplet sit for 30 minutes (Table 4). It is important to note that unlike the silylated samples, paper samples that were not silylated absorbed water instantly, and therefore recorded a contact angle of $0^{\circ}$.

\section{DISCUSSION}

From the data collected, it can be seen that the hydrophobicity of all paper samples, evidenced by contact angle, gradually declines over time in all solutions, but this is especially evident in Coca-Cola and in coffee (Figure $6 \mathrm{C}$ and $\mathrm{H}$ ). Given that the paper was made hydrophobic through silylation, it is expected that the decrease in hydrophobicity observed is due to the acidic properties of these solutions which may catalyze the hydrolysis of silane substituents from cellulose, releasing the corresponding silanol into solution. On the other hand, samples exposed only to water showed minimal decline in contact angle, indicating no change in hydrophobicity as a result. Furthermore, it was found that there was no significant change in the mass of samples before and after exposure to water for 6 hours (Table 3), suggesting that minimal leaching occurred in water. Additionally, the contact angles of these samples, having slowly declined throughout initial testing due to wetting, had been almost completely restored to their original values after being dried for a sufficiently long time (approximately 12 hours). This suggests that the decline in contact angle was instead primarily due to wetting. Samples exposed to CocaCola exhibited permanent loss of hydrophobicity, as contact angles were not completely restored to their initial values after sufficient drying (Table 3). As such, with Coca-Cola, and all other drinks, the magnitude of decline in contact angle is likely due to a combination of both wetting and desilylation. Importantly, of the eight liquids, six were acidic (Coca-Cola, coffee, orange-juice, sprite, apple-juice, and vodka) and these were the liquids that caused the most significant changes in hydrophobicity. Thus, it is possible that the acidic properties contributed to the removal of the silane from the paper, accounting for the loss in hydrophobicity. 

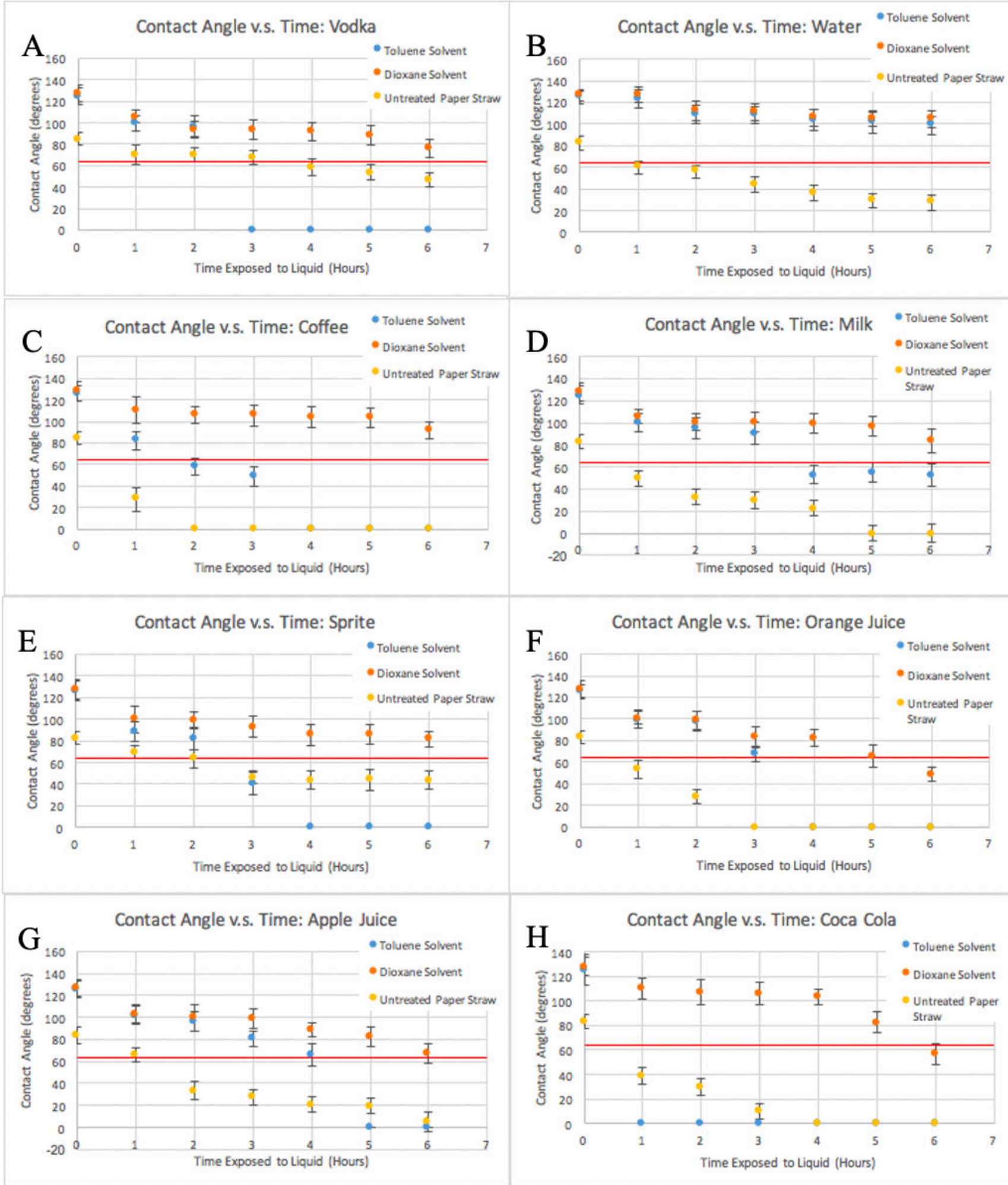

Figure 6. Mean calculated contact angle of $5 \mu \mathrm{l}$ water droplet on paper samples. Comparisons were made between untreated paper, samples silylated using toluene, and samples silylated with dioxane. Errors in the x-axis were omitted as the relative uncertainty for the time measurement was negligible $(<0.5 \%)$. The red line in each of the contact angle vs. time graphs projects the contact angle of current plastic straws at $64^{\circ}$, where errors were omitted for clarity of comparison and points at each hour were fitted. Error bars represent 1 standard deviation of 3 contact angle measurements for each of the 4 samples in all graphs. P-values were found using a single factor ANOVA test. Each figure illustrates contact angles after exposure to A. vodka $(p=0.045)$, B. water $(p<0.001)$ where $t^{\prime}=0.567$ between the treated straws, C. coffee $(p<0.001)$, D. milk $(p<0.001)$, E. sprite $(p=0.028)$, F. orange juice $(p=0.029)$, G. apple juice $(p=0.018), H$. Coca-Cola $(p=0.018)$. Sample size of all treatments was $n=4$. 
Table 2. Comparison of change in mass after silylation of samples where the average mass per sample for all treatment groups ranged from $9.81 \mathrm{mg}$ to $11.2 \mathrm{mg}$.

\begin{tabular}{|l|l|}
\hline Treatment & Average Change in Mass per Sample $( \pm 0.029 \mathrm{mg})$ \\
\hline DSS & 0.23 \\
\hline TSS & 0.19 \\
\hline DS & 0.023 \\
\hline TS & 0.0049 \\
\hline
\end{tabular}

Table 3. Comparison of change in the contact angle and mass of samples after exposure to water and Coca Cola for 6 hours and sufficient drying. For all groups, $n=5$. $t^{\prime}$-scores between initial and final contact angles were calculated for $D S S$ in water $\left(t^{\prime}=0.194\right)$, TSS in water $\left(t^{\prime}=\right.$ 0.414), DSS in Coca-Cola ( $\left.t^{\prime}=2.423\right)$ and TSS in Coca-Cola $\left(t^{\prime}=128.8\right)$. *Samples were observed to be tinted brown after exposure to Coca-Cola and sufficient drying.

\begin{tabular}{|l|c|c|c|c|l|}
\hline \multicolumn{2}{|l|}{ Treatment } & $\begin{array}{l}\text { Initial Contact } \\
\text { Angle (degrees) }\end{array}$ & $\begin{array}{l}\text { Final Contact } \\
\text { Angle (degrees) }\end{array}$ & $\begin{array}{l}\text { Initial Mass } \pm \\
0.029 \mathrm{mg})\end{array}$ & $\begin{array}{l}\text { Final Mass ( } \pm \\
0.029 \mathrm{mg})\end{array}$ \\
\hline \multirow{2}{*}{ Water } & DSS & $128.2 \pm 7.1$ & $126.3 \pm 6.7$ & 41.7 & 41.7 \\
\cline { 2 - 6 } & TSS & $126.9 \pm 7.8$ & $122.0 \pm 8.9$ & 38.2 & 38.3 \\
\hline Coca-Cola & DSS & $129.1 \pm 6.4$ & $99.5 \pm 10.4$ & 41.1 & $45.8^{\star}$ \\
\cline { 2 - 6 } & TSS & $128.8 \pm 9.3$ & 0 & 39.5 & $45.5^{\star}$ \\
\hline
\end{tabular}

Table 4. Comparison of change in contact angle of drops before and after sitting on DSS and TSS samples for 30 minutes. ( $n=3$ for both groups, $t^{\prime}=3.027$ between the final contact angles of the TSS and DSS samples).

\begin{tabular}{|l|l|l|}
\hline Treatment & Initial Contact Angle measurement $\left(^{\circ}\right)$ & $\begin{array}{l}\text { Final Contact Angle (after 30 minutes) } \\
\left({ }^{\circ}\right)\end{array}$ \\
\hline DSS & $128.4 \pm 6.9$ & $120.8 \pm 8.1$ \\
\hline TSS & $128.1 \pm 7.5$ & $83.7 \pm 9.2$ \\
\hline
\end{tabular}


Following the silylation of the paper samples, the change in mass upon the addition of the silane was only $2.4 \%$. Given the fractional increase in mass, it is reasonable to conclude that only a small amount of silane was attached to the paper. As such, this would not likely impact the inherent biodegradability of the paper itself.

Comparisons between DSS and TSS treatments show that TSS samples experience a significantly faster decline in hydrophobicity than the DSS samples with respect to their measured contact angles. TSS samples showed a complete loss of hydrophobicity within the 6-hour exposure in every liquid except for milk and water (Figure 6). In contrast, DSS samples, although showing some decline, remained fairly hydrophobic after exposure to each liquid, with the lowest contact angle observed being just under $50^{\circ}$ for orange juice. Furthermore, mass correlations were performed to compare the loading of silane between DSS and TSS samples. It was found that DSS samples increased in mass by $0.225 \mathrm{mg}$ per sample following silylation, which is approximately $18 \%$ more than the $0.1905 \mathrm{mg}$ increase per sample seen in TSS samples. Because the procedure used in preparation ensured that there was a negligible amount of solvent remaining on the samples, the differences in the two treatments were likely caused by variance in the loading of silane to the samples. While an $18 \%$ increase seems small, it corresponds to a difference of over 6x1016 molecules, meaning there was a substantial increase in the number of silane molecules binding to samples treated in dioxane as compared to toluene as the solvent.

Long-term contact angle measurements (Table 4) show that drops were absorbed into TSS samples at a faster rate than DSS samples. To explain this, it is postulated that there is an enhanced effect of silane derivatization when using dioxane as the solvent during the preparation phase, rather than toluene. This is most likely to do with the reaction rate. During reaction the 6 ' hydroxyl of the cellulose subunit likely attacks the silane, ejecting the chlorine as a leaving group and forming a polar silylated cellulose transition state. Because dioxane (4.8 on polarity index) is much more polar than toluene (2.4 on the polarity index), it may lead to better stabilization of the polar transition state, lowering the energy required for the reaction to proceed. This would result in an increased reaction rate and more silylation throughout the reaction period (13). In contrast, toluene is less capable of this, and would therefore have a lower reaction rate causing less derivatization on TSS-treated paper.
Contact angle measurements indicate that DSS samples are significantly ( $p<0.05$ for all tests) more hydrophobic than both commercial paper straws, and plastic straws (Figure 6). Although the hydrophobicity declines with time, it took at least 5 hours in each drink for the contact angle to reach that of the plastic straw $\left(64^{\circ}\right)$. In a restaurant setting, it was found that the average time to drink a soda is less than 15 minutes (14). As such, one can assume that most straws are typically only in use for 2 or fewer hours, and thus the silylated paper can be an adequate replacement for plastic straws. Furthermore, treated samples maintain hydrophobicity to a greater degree than current paper straws, which is notable considering that the paper straws are much more dense than the filter paper used in this experiment. Filter paper is extremely absorbent (0.1 $\pm 0.029 \mathrm{~s}$ for a $1 \mathrm{~cm}^{2}$ paper to completely absorb $5 \mu \mathrm{L}$ water); treating paper with greater structural integrity would not only provide an appealing rigidity to consumers but further enhance the ability of samples to maintain hydrophobicity, as it would be less susceptible to wetting.

The molecule that is hydrolyzed from the paper in acidic conditions is likely dimethyloctadecylsilanol. Because the samples were thoroughly washed with methanol, only a negligible amount of solvent is expected to remain on the paper, so it is probable that the leaching of dimethyloctadecylsilanol would be the source of any hazard. While hazard statements for this molecule are unavailable, similar molecules were reviewed to provide indication of relevant risks and warnings. For instance, triethylsilanol is flammable but non-toxic (Figure $7(A)$ ), tertbutyldimethylsilanol is both flammable and toxic (Figure $7(B))(15,16)$, and octadecylsilane has a toxicity warning (Figure $7(C)$ ) (17). Due to the structural similarities between these compounds, it is reasonable to assume that dimethyloctadecylsilane (Figure 7(D)) may confer similar risks, however, there is no evidence to confirm this.

Overall, this experiment was well designed, but did exhibit limitations. Due to a lack of time and resources, the toxicity and biodegradability of treated paper was not assessed. Likewise, investigations into the physical use of treated paper as a straw were not carried out, as no physical prototype was created. Furthermore, filter paper was used for testing rather than the paper that is currently used in paper straws. During measurement of contact angle, an optical tensiometer for contact angle analysis was not employed, but rather a high definition camera and stand was used. Thus, future investigations should seek to address these issues to 


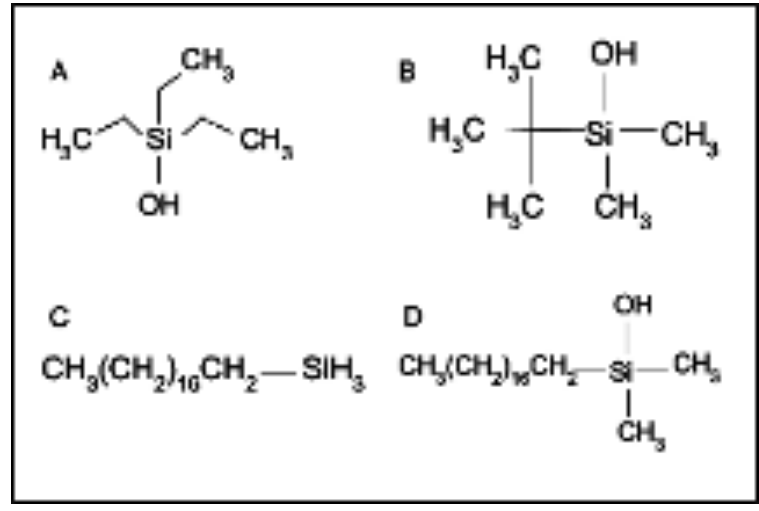

Figure 7. Chemical structure of (A) triethylsilanol, tert-butyldimethylsilanol, dimethyloctadecylsilanol. of (A) triethylsilano

\section{CONCLUSION}

Ultimately, the hydrophobization of paper through silylation of cellulose was successfully accomplished through this methodology, where samples treated with dioxane provided the most robust results over time and during exposure to different beverages. Nonetheless, all treated samples demonstrated considerably greater retention of hydrophobicity than current paper straws. Moreover, the altered paper structure is likely to be biodegradable, accounting for any environmental concerns.

Further investigations are required to determine the exact effects of the leached dimethyloctadecylsilanol and its potential hazards. Experimentation should also explore different reaction times and solvents to improve silylation efficiency confer sustained hydrophobicity. In addition, using a slightly altered reagent and different solvents could potentially further reduce the toxicity of the product. Overall, silylation is a promising method for the hydrophobization of paper and holds great potential towards improving the structural integrity of paper straws.

\section{ACKNOWLEDGEMENTS}

We would like to thank Dr. John Sherman for his contributions as our mentor, Dr. Guillaume Bussiere for allowing us to use his analytical balances, as well as the Brumer lab for use of their lab spaces, and Dr. Laleh Solhi for her help with preparation of samples.

\section{REFERENCES}

1. Gourmelon G. Global plastic production rises, recycling lags. [Internet]. Washington, DC: Worldwatch Institute; 2015 [cited 2019 May 25]. Available from: http://www.plastic-resource-center.com/wpcontent/uploads/2018/11/Global-Plastic-ProductionRisesRecycling-Lags.pdf

2. Andrady AL. Plastics and the environment. [Internet]. USA: John Wiley \& Sons, Inc.; 2005. Chapter 1, An Environmental Primer. [cited 2019 May 25]. doi:10.1002/0471721557.ch1

3. Fugetsu B, Sano E, Sunada M, Sambongi Y, Shibuya T, Wang $X$, et al. Electrical conductivity and electromagnetic interference shielding efficiency of carbon nanotube/ cellulose composite paper. Carbon. 2008;46(9):12561258. doi:10.1016/j.carbon.2008.04.024

4. Brown, LeMay, Bursten, Murphy, Woodward. Chemistry: The central science [Internet]. 11th edition. Upper Saddle River, NJ: Pearson Prentice Hall; 2009. Chapter 13.3, Factors affecting solubility. [cited 2019 May 25]. Available from: http://wps.prenhall.com/wps/media/ objects/3312/3391718/blb1303.html

5. Map: chemistry - The central science (Brown et al.). [Internet]. Chemistry LibreTexts; 2020. Chapter 8.8, Strength of Covalent Bonds. [cited 2019 May 25]. Available from: https://chem.libretexts.org/Bookshelves/ General_Chemistry/Map\%3A_Chemistry_-_The_Central_ Science_(Brown_et_al.)/08._Basic_Concepts_of_Chemical Bonding/8.8\%3A_Strength_of_Covalent_Bonds

6. Jain RK, Agnish SL, Lal K, Bhatnagar HL. Reactivity of hydroxyl groups in cellulose towards chlorotri(p-tolyl) methane. Makromol. Chem. 1985;186(12):2501-2512. doi:10.1002/macp.1985.021861210

7. Yuan Y, Lee TR. Contact angle and wetting properties. [Internet]. In: G. Bracco, B. Holst, editors. Surface Science Techniques. Berlin, Heidelberg: Springer. 2013. p. 3-34. (Springer Series in Surface Sciences, vol 51). doi:10.1007/978-3-642-34243-1_1

8. Ortega F, Velez E, Somanathan R. Synthesis and use of reverse-phase silica gel for HPLC in undergraduate chemistry. J. Chem. Educ. 1996;73(2):A26. doi:10.1021/ ed073pa26

9. GE Healthcare Whatman ${ }^{\mathrm{TM}}$ Qualitative Filter Paper: Grade 1 Circles [Internet]. Thermo Fisher Scientific. [cited 2019 May 25]. Available from: https://www.fishersci.ca/shop/ products/whatman-qualitative-grade-plain-circles-grade$1-19 / p-2428689$

10. Chloro(dimethyl)octadecylsilane [Internet]. Canada: Sigma-Aldrich. [cited 2019 Jul 10]. Available from: https:// www.sigmaaldrich.com/catalog/product/aldrich/289108

11. Boonniteewanich J, Pitivut S, Tongjoy S, Lapnonkawow S, Suttiruengwong S. Evaluation of carbon footprint of bioplastic straw compared to petroleum based straw products. Energy Procedia. 2014;56:518-524. 
doi:10.1016/j.egypro.2014.07.187

12. Kranias S. Effect of drop volume on static contact angles [Internet]. Hamburg, Germany: KRÜSS GmbH. Available from: https://www.kruss-scientific.com/fileadmin/user upload/website/literature/kruss-tn310-en.pdf

13. Polarity index [Internet]. Polymer Analysis Laboratory, Louisiana State University. [cited 2019 May 25]. Available from: http://macro.Isu.edu/howto/solvents/Polarity\%20 index.htm

14. McElrea $\mathrm{H}$, Standing L. Fast music causes fast drinking. Percept Mot Skills. 1992;75(2):362. doi:0.2466/ pms.1992.75.2.362

15. Triethylsilanol 380423 [Internet]. Sigma-Adrich. [cited 2019 May 25]. Available from: https://www.sigmaaldrich. com/catalog/product/aldrich/380423?lang=en ®ion

16. tert-Butyldimethylsilane 294764 [Internet]. SigmaAdrich. [cited 2019 May 25]. Available from:

https://www.sigmaaldrich.com/catalog/product/ aldrich/294764?lang=en®ion

17. Octadecylsilane [Internet]. Canada: Sigma-Aldrich. [cited 2019 May 25]. Available from: https://www.sigmaaldrich. com/catalog/product/aldrich/442291

18. O'Kane JM, Sherrington DC. Hysteresis-like behavior in the swelling/deswelling of polystyrene crosslinked resins using binary solvent mixtures. Macromolecules. 1990;23(25):5286-5291. doi:10.1021/ma00227a018

\section{APPENDIX}

Analyses excluded samples treated with dimethylformamide (DMF) due to the specific reaction that occurred in the presence of a combination of DMF and chlorosilane with the paper. Approximately 20 minutes into the reaction, samples appeared to be degrading in the solution. Post-reaction samples demonstrated severe, irreversible swelling; the paper squares had expanded into cubes (Figure 8). This phenomenon is potentially caused by hysteresis, which is the process in which a physical property changes slower than the effect that causes it. In this case, it is possible that the initially solvent-filled, swollen paper was effectively sealed before deswelling could complete, thus, accounting for the vast increase in mass that was measured as well as the altered size and shape of the samples (18).

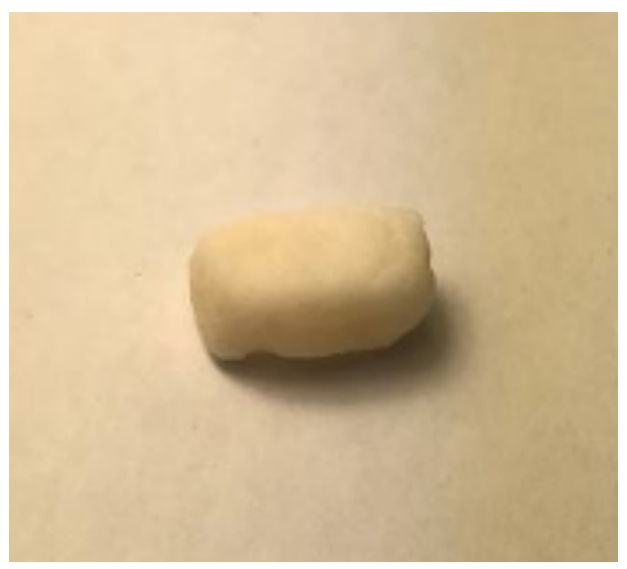

Figure $8.1 \mathrm{~cm}^{2}$ FSS sample, which became swollen following reaction with the FSS solvent and reagent mixture. The mass of the FSS samples significantly increased following exposure, and a gel matrix was expelled upon making an incision in these samples. 